\title{
Synthesis of 4-(2-Hydroxy-5-methylphenyl)-1,3-Dithiol-2-ylidene Derivatives
}

\author{
CATALIN NECULAI LUNGU ${ }^{1}$, IULIEAN VASILE ASAFTEI ${ }^{1}$, ION SANDU ${ }^{2,3}$, PAUL CHIRITA ${ }^{4}$, LUCIAN GABRIEL BAHRIN ${ }^{1,5 *}$ \\ ${ }^{1}$ Alexandru Ioan Cuza University of lasi, Department of Chemistry, 11 Carol I Blvd., 700506, lasi, Romania \\ ${ }^{2}$ Alexandru Ioan Cuza University of lasi, Arheoinvest Platform, Scientific Investigation Laboratory, 11 Carol I Blvd., 700506, Iasi, \\ Romania \\ ${ }^{3}$ Romanian Inventors Forum, 3 Sf. Petru Movila Str., Bloc L11, III/3, 700089, Iasi, Romania \\ ${ }^{4}$ University of Craiova, Department of Chemistry, 107l Calea Bucuresti, 200478, Craiova, Romania \\ ${ }_{5}^{5}$ Petru Poni Institute of Macromolecular Chemistry, 41 A Grigore Ghica Voda Alley, 700487, Iasi, Romania
}

The reaction of 4-(2-hydroxy-5-methylphenyl)-1,3-dithiolium perchlorates with various methylene active compounds has provided the corresponding 1,3-dithiol-2-ylidene derivatives. The reactions have been performed under basic conditions using DBU. The newly obtained derivatives were characterized by NMR, MS spectrometry and IR spectroscopy.

Keywords: acetophenones, 1,3-dithiolium salts, methylene active, ylidenes

The development of new organic superconductors is a major topic in the field of molecular conductors [1]. For many years, all the organic $\delta$-electron donors with conductivity properties were limited to the tetrachalcogenafulvalenes compounds [2-4]. Recently, the nontetrachalcogenofulvalenes containing a 1,3-dithiol-2ylidene based $\delta$-donor unity proved to give superconducting salts [5]. Heterocycles are important compounds for the material chemistry and not only. Amongst these, sulphur and nitrogen-containing heterocycles receive a great deal of attention [6-17]. These types of compounds have also biological applications and notonly (e.g. daily life and even the educational process) [18-29]. Charge-transfer [30-41] or push-pull [42-55] compounds have important applications in the field of conducting materials. An important precursor for 1,3-dithiol-2-ylidene derivatives are the 1,3-dithiolium-2-yl compounds [56, 57]. 1,3-Dithiolium salts contain a positive charge located at the $\mathrm{C}(2)$ position and consequently these systems are prone to nucleophilic interactions at this position [58, 59].

We are reporting here the synthesis of novel 4-(2hydroxy-5-methylphenyl)-1,3-dithiol-2-ylidene derivatives from the corresponding 1,3-dithiolium salts and various methylene active compounds.

\section{Experimental part}

\section{Analysis methods}

Melting points were obtained on a KSPI melting-point meter and are uncorrected. IR spectra were recorded on a Bruker Tensor 27 instrument. NMR spectra were recorded on a Bruker $300 \mathrm{MHz}$ spectrometer. Chemical shifts are reported in ppm downfield from TMS. Mass spectra were recorded on a Thermo Scientific ISQ LT instrument.

\section{Synthesis}

Following the reported experimental procedure for dithiocarbamates $\mathbf{3}$ and 1,3-dithiolium perchlorates $\mathbf{4}$ we have synthesized them as described in scheme 1 [60]. 1,3-Dithiol-2-ylidene derivatives 6a-d were synthesized according to the conditions presented in scheme 2.

1,3-Dithiol-2-ylidene derivative 6a; General Procedure

To a suspension of 1,3-dithiolium perchlorate $\mathbf{4 d}(0.4 \mathrm{~g}$, $1 \mathrm{mmol}$ ) in acetonitrile $(15 \mathrm{~mL})$ 2,4-pentandione (5a, $0.1 \mathrm{~mL}, 1 \mathrm{mmol}$ ) was added and the reaction mixture was brought to $60^{\circ} \mathrm{C}$. DBU $(0.34 \mathrm{~mL}, 2.2 \mathrm{mmol})$ was then added and the reaction was heated for $4 \mathrm{~h}$ under stirring. The solution was then poured into water $(100 \mathrm{~mL})$ and concentrated hydrochloric acid $(3 \mathrm{~mL})$ was added. The precipitate was filtered under vacuum and recrystallized from ethanol; yield $0.21 \mathrm{~g}(65 \%)$.

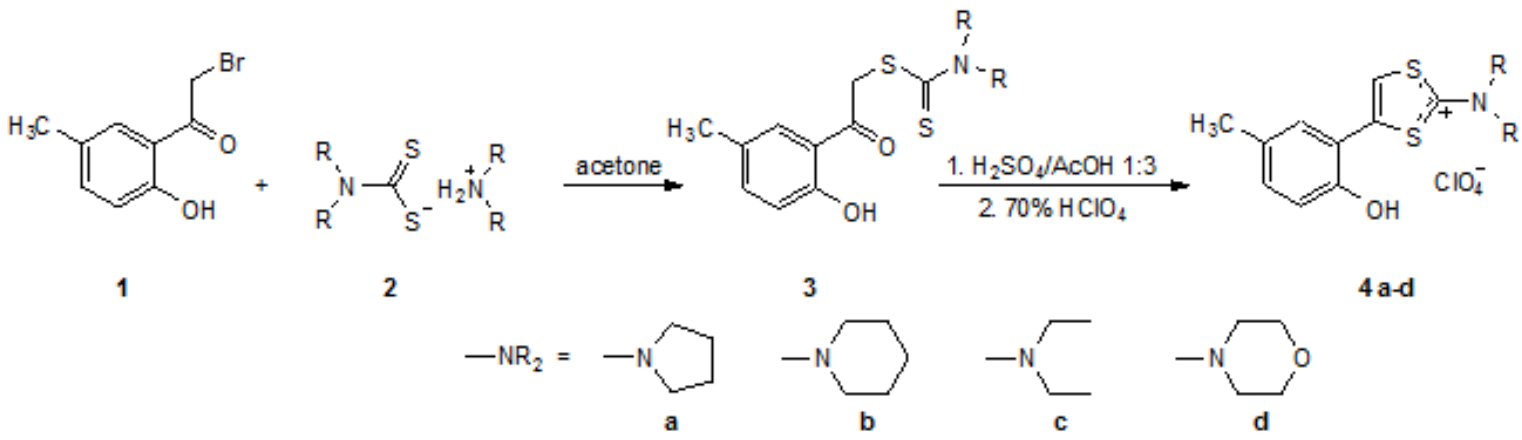

Scheme 1. Synthesis of dithiocarbamates 3 and 1,3-dithiolium perchlorates 4 
<smiles>[R]C(=O)C(C([R1])=O)=C1SC=C(c2cc(C)ccc2O)S1</smiles>

4

5

$6 a-d$

\begin{tabular}{|c|c|c|}
\hline 5,6 & $\mathrm{R}^{1}$ & $\mathrm{R}^{2}$ \\
\hline $\mathbf{a}$ & $\mathrm{CH}_{3}$ & $\mathrm{CH}_{3}$ \\
\hline $\mathbf{b}$ & $\mathrm{CH}_{3}$ & $\mathrm{OEt}$ \\
\hline c & & \\
\hline d & & \\
\hline
\end{tabular}

Scheme 2. Synthesis of 1,3-dithiol2-ylidene derivatives $6 a-d$

Table 1

ANALYTICAL AND SPECTRAL DATA OF 1,3-DITHIOL-2-YLIDENE DERIVATIVES 6a-d

\begin{tabular}{|c|c|c|c|c|}
\hline & M.p., ${ }^{\circ} \mathrm{C}$ & $\eta, \%$ & IR-ATR, $\mathrm{cm}^{-1}$ & NMR, ppm \\
\hline $6 a$ & $121-122$ & 65 & $\begin{array}{c}2938,2861,1638 \\
1558,1422,1322 \\
1144,951\end{array}$ & 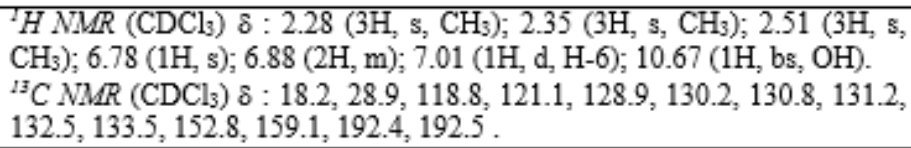 \\
\hline $6 \mathrm{~b}$ & $101-102$ & 51 & $\begin{array}{c}2944,1641,1638, \\
1574,1431,1241, \\
1026,918,678\end{array}$ & 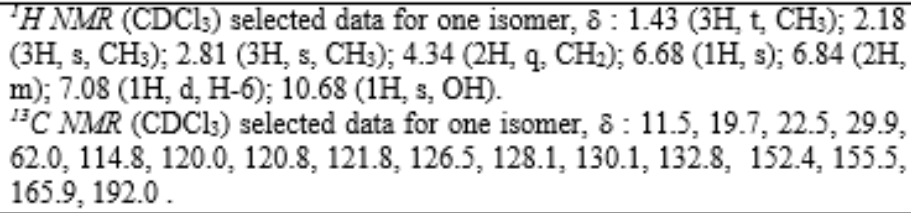 \\
\hline $6 c$ & $131-132$ & 54 & $\begin{array}{c}2878,1638,1567, \\
1469,1208,810,640\end{array}$ & $\begin{array}{l}{ }^{I} \mathrm{H} \mathrm{NMR}_{\left(\mathrm{CDCl}_{3}\right)} 8: 2.21\left(3 \mathrm{H}, \mathrm{s}, \mathrm{CH}_{3}\right) ; 6.88(1 \mathrm{H}, \mathrm{bs}) ; 7.57(2 \mathrm{H}, \mathrm{m}) ; 7.76 \\
(4 \mathrm{H}, \mathrm{m}) ; 7.49(1 \mathrm{H}, \mathrm{s}, \mathrm{H}-4) ; 10.07(1 \mathrm{H}, \mathrm{bs}, \mathrm{OH}) . \\
{ }^{13} \mathrm{C} N M R\left(\mathrm{CDCl}_{3}\right) 8: 14.4,120.8,123.1,124.9,125.1,126.3,128.1,129.0, \\
133.0,134.4,135.4,136.4,139.5,140.1,151.8,166.0,187.8,188.2\end{array}$ \\
\hline $6 \mathrm{~d}$ & $124-125$ & 56 & $\begin{array}{c}2918,1567,1361 \\
\quad 840,685\end{array}$ & 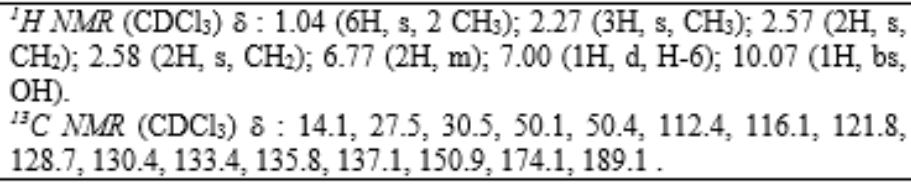 \\
\hline
\end{tabular}

\section{Results and discussions}

The phenacyl carbodioates 3 were synthesized according to the reported procedure [60] by reacting 2bromo-1-(5-methyl-2-hydroxyphenyl) ethan-1-one (1) with pyrrolidinium pyrrolidine-1-carbodithioate (2a), piperidinium piperidine-1-carbodithioate (2b), sodium $N, N$ diethyldithiocarbamate (2c) and morpholinium morpholine-4-carbodithioate (2d), in acetone under heating (scheme 1). The later ammoniun salts were synthesized by the reaction of secondary amine with carbon disulfide [61].

Acid catalyzed cyclization of phenacyl carbodithioates 3a-c provided is known to provide the corresponding 1,3dithiol-2-ylium cations $[62,63]$, which where isolated as salts of perchloric acid $\mathbf{4 a - d}$. The analytical and spectral data of compound $\mathbf{3}$ and $\mathbf{4}$ are in agreement with those previously reported [60].

Due to the positive charge located at the $C(2)$ position, 1,3-dithiol-2-ylium ring readily interact with nucleophiles [9]. The target 1,3-dithiol-2-ylidene derivatives 6 have been obtained from the reaction of 1,3-dithiolium perchlorates
4 with carbanions derived from various methylene active compounds $\mathbf{5}$ (scheme 2). The $C$-nucleophiles have been generated using 1,8-diazabicyclo[5.4.0]undec-7-ene (DBU) as base (but week nucleophile) to extract a proton from the active methylene moiety. The reactions have been performed using $2.2 \mathrm{eg}$. of DBU, in acetonitrile, at $60^{\circ} \mathrm{C}$ providing 1,3-dithiol-2-ylidene derivatives 6 in good isolated yields (table 1). Depending on the substitution at the C-2 of 1,3-dithiolium ring the yields remains in the same range ( $\pm 2-3 \%)$.

The formation of 1,3-dithiol-2-ylidene derivatives $6 \mathbf{a}$-d is supported by analytical and spectral data (table 1). IR spectroscopy indicates the presence of new carbonyl or ester bands, which come from the active methylene compounds. ${ }^{1} \mathrm{H}$ NMR spectra reveals the presence of new methyl/methylene aliphatic signals for derivatives 6a-d.

${ }^{13} \mathrm{C}$ NMR spectra indicates the disappearance of the signal of the positively charged $\mathrm{C}(2)$ atom and the appearance of the new signals corresponding to the new double bonded carbon atoms. Also, the new signals 
corresponding to the carbonyl groups and ester groups confirm the structures of the new 1,3-dithiol-2-ylidene derivatives. The compound $\mathbf{6} \boldsymbol{b}$ has been obtained as a mixture of two isomers due to the asymmetry of ethyl acetoacetate. The spectral data are presented only for one isomer. Mass spectrometry analysis has also confirmed providing the following molecular peaks: $306\left(\mathrm{C}_{15} \mathrm{H}_{14} \mathrm{O}_{3} \mathrm{~S}_{2}\right)$ for $\mathbf{6 a}, 336\left(\mathrm{C}_{16} \mathrm{H}_{10} \mathrm{O}_{4}\right)$ for $\mathbf{6 b}, 352\left(\mathrm{C}_{19} \mathrm{H}_{12} \mathrm{O}_{3} \mathrm{~S}_{2}\right)$ for $6 \mathbf{c}$, and $346\left(\mathrm{C}_{18} \mathrm{H}_{18} \mathrm{O}_{3} \mathrm{~S}_{2}\right)$ for $6 \mathrm{~d}$.

\section{Conclusions}

The synthesis of new 4-(2-hydroxy-5-methylphenyl)-1,3dithiol-2-ylidene derivatives has been performed by reacting the corresponding 1,3-dithiolium perchlorates with various active methylene compounds under basic conditions (DBU).

\section{References}

1.ISHIGURO, T., YAMAII, K., SAITO, G., Organic Superconductors (Springer Series in Solid-State Sciences), $2^{\text {nd }}$ edition, Ed. FULDE, P., Springer, Berlin, vol. 88, 1998.

2.ADACHI, T., OJIMA, E., KATO, K., KOBAYASHI, H., MIYAZAKI, T., TOKUMOTO, M., KOBAYASHI, A., J. Am. Chem. Soc., 122, 2000, p. 3238.

3.OTSUBO, T., TAKIMIYA, K., Bull. Chem. Soc. J pn., 77, 2004, p. 43. 4.YAMADA, J., SUGIMOTO, T., TTF Chemistry- Fundamentals and Applications of Tetrathiafulvalene, Kodansha-Springer, 2004.

5.YAMADA, J.-i., J. Mater. Chem., 14, 2004, p. 2951.

6.HRIB, C.G., CHIRITA, P., SANDU, I.G., ASAFTEI, I.V., SARBU, L.G., EARAR, K., Rev. Chim. (Bucharest), 66, no. 7, 2015, p. 983.

7.SELIGER, H., HAPP, E., CASCAVAL, A., BIRSA, M.L., NICOLAESCU, T., POINESCU, I., COJ OCARIU, C., Eur. Polym. J., 35, 1999, p. 827.

8.BRAVERMAN, S., CHERKINSKY, M., BIRSA, M.L., TICHMAN, S., GOLDBERG, I., Tetrahedron Lett., 42, 2001, p. 7485.

9.BIRSA, M. L., Synth. Commun., 32, 2002, p. 115.

10.BRAVERMAN, S., CHERKINSKY, M., BIRSA, M.L., ZAFRANI, Y., Eur. J. Org. Chem., 2002, p. 3198.

11.BIRSA, M.L., CHERKINSKY, M., BRAVERMAN, S., Tetrahedron Lett., 43, 2002, p. 9615.

12.LEVI, M.D., GOFER, Y., CHERKINSKY, M., BIRSA, M.L., AURBACH, D., BERLIN, A., Phys. Chem. Chem. Phys., 5, 2003, p. 2886.

13. CHIRITA, P., HRIB, C.G., SANDU, I., LUNGU, N.C., SARBU, L.G., EARAR, K., Rev. Chim. (Bucharest), 66, no. 8, 2015, p. 1151.

14.BELEI, D., BICU, E., JONES, P. G., BIRSA, M. L., Synlett, 2010, p. 931.

15. BELEI, D., BICU, E., JONES, P. G., BIRSA, M.L., J. Heterocycl. Chem., 48, 2011, p. 129.

16.BELEI, D., ABUHAIE, C., BICU, E., JONES, P. G., HOPF, H., BIRSA, M.L., Synlett, 23, 2012, p. 545.

17.HOPF, H., JONES, P.G., NICOLESCU, A., BICU, E., BIRSA, M.L., BELEl, D., Chem. Eur. J., 20, 2014, p. 5565.

18.GOSAV, S., PRAISLER, M., BIRSA, M.L., Int. J. Mol. Sci., 12, 2011, p. 6668.

19.BIRSA, M.L., SANDU, I., BAHRIN, L.G., Rev. Chim. (Bucharest), 65, no. 2, 2014, p. 174.

20. BABII, C., MIHALACHE, G., BAHRIN, L.G., NEAGU, A.-N., GOSTIN, I., MIHAI, C.T., SARBU, L.G., BIRSA, M.L., STEFAN, M., PLOS ONE, 13, no.4, 2018, p. e0194898.

21.BAHRIN, L.G., APOSTU, M.O., BIRSA, M.L., STEFAN, M., Bioorg. Med. Chem. Lett., 24, 2014, p. 2315.

22.GOSAV, S., BIRSA, M.L., Rom. Rep. Phys., 66, 2014, p. 411.

23.BAHRIN, L.G., HOPF, H., J ONES, P.G., SARBU, L.G., BABII, C., MIHAI, A.C., STEFAN, M., BIRSA, M.L., Beilstein J. Org. Chem., 12, 2016, p. 1065.

24.SARBU, L.G., LUNGU, C.N., BALAN, A., BAHRIN, L.G., Rev. Chim. (Bucharest), 65, no. 10, 2014, p. 1135.
25.SARBU, L.G., APOSTU, M.O, SANDU, I., BAHRIN L.G., MANEA. L.R., Rev. Chim. (Bucharest), 65, no. 11, 2014, p. 1327.

26.BAHRIN, L.G., SARBU, L.G., HOPF, H., JONES, P.G., BABII, C., STEFAN, M., BIRSA, M.L., Bioorg. Med. Chem., 24, 2016, p. 3166.

27.BAHRIN, L.G., ASAFTEI, I.V., SANDU, I., SARBU, L.G., Rev. Chim. (Bucharest), 65, no. 9, 2014, p. 1046.

28. ANDRONACHE, D., BOCOS, M., NECULAU, B.C., Procedia Soc. Behav. Sci., 180, 2015, p. 715.

29.SANDU, C., NECULAU, B.C., J. Pub. Admin. Fin. L., 6, 2014, p. 198. 30. MEZIERE, C., ALLAIN, M., OLIVERAS-GONZALEZ, C., CAUCHY, T., VANTHUYNE, N., SARBU, L.G., BIRSA, M.L., POP, F., AVARVARI, N., Chirality, 30, 2018, p. 568.

31.YAMADA, J.-i., J. Mater. Chem., 14, 2004, p. 2951.

32. WADA, A., NISHIDA, J.-i., MAITANI, M.M., WADA, Y., YAMASHITA, Y., Chem. Lett., 43, 2014, p. 296.

33.SARBU, L.G., HRIB, C.G., BIRSA, M.L., Acta Cryst., E69, 2013, p. 01169.

34.SARBU, L.G., LUNGU, N.C., ASAFTEI, I.V., SANDU, I., BIRSA, M.L., Rev. Chim. (Bucharest), 65, no. 3, 2014, p. 325.

35.BUHACEANU, R., LUNGU, N.C., FORNA, N.C., ASAFTEI, I.V., CHIRITA, P., BIRSA, M.L., Rev. Chim. (Bucharest), 64, no. 8, 2013, p. 802.

36.SARBU, L.G., BAHRIN, L.G., Acta Chem. Iasi, 21, 2013, p. 47.

37.CHIRITA, P., ASAFTEI, I.V., SANDU, I., SARBU, L.G., LUPU, V.V., Rev. Chim. (Bucharest), 68, no. 1, 2017, p. 147.

38. BIRSA, M.L., GANJU, D., J. Phys. Org. Chem., 16, 2003, p. 207.

39. BIRSA, M.L., Synth. Commun., 33, 2003, p. 3071.

40. SARBU, L.G., BIRSA, M.L., Acta Chem. lasi, 19, 2011, p. 125.

41. BIRSA, M.L., ASAFTEI, I.V., Monat. Chem., 139, 2008, p. 1433.

42. SARBU, L.G., BIRSA, A., HOPF, H., BIRSA, M.L., Phosphorus, Sulfur, and Silicon, and the Related Elements, 186, 2011, p. 1246.

43. SARBU, L.G., BIRSA, A., IGNAT, L., HOPF, H., BIRSA, M.L., Acta Chem. Iasi, 18, 2010, p. 71.

44. BIRSA, M.L., J ONES, P.G., BRAVERMAN, S., HOPF, H., Synlett, 2005, p. 640.

45. BIRSA, M.L., J ONES, P.G., HOPF, H., Eur. J. Org. Chem., 2005, p. 3263.

46. BIRSA, M.L., HOPF, H., Synlett, 2007, p. 2753.

47. BIRSA, M.L., HOPF, H., Synlett, 2009, p. 3000.

48. BIRSA, M.L., HOPF, H., Heteroatom Chem., 21, 2010, p. 126.

49. BIRSA, M.L., J ONES, P.G., HOPF, H., Synlett, 2011, p. 259.

50. SARBU, L.G., HOPF, H., GRUENENBERG, J., BIRSA, M.L., Synlett, 26, 2015, p. 87.

51. SARBU, L.G., BICU. E., HOPF, H., BIRSA, M.L., Rev. Chim. (Bucharest), 65, no. 4, 2014, p. 398.

52.SARBU, L.G., HOPF, H., JONES, P.G., BIRSA, M.L., Beilstein J. Org. Chem., 10, 2014, p. 2550.

53.BAHRIN, L.G., SARBU, L.G., JONES, P.G., BIRSA, M.L., HOPF, H., Chem. Eur. J., 23, 2017, p. 12338.

54.PAVEL, S., HOPF, H., J ONES, P.G., ASAFTEI, I.V., SARBU, L.G., BIRSA, M.L., Monat. Chem., 147, 2179 (2016).

55. SARBU, L.G., BIRSA, A., HOPF, H., BIRSA, M.L., Acta Chem. Iasi, 18, 2010, p. 186.

56.DIRTU, D., LUNGU, N.C., CHIRITA, P., SANDU, I.G., BIRSA, M.L., EARAR, K., SARBU, L.G., Rev. Chim. (Bucharest), 67, no. 3, 2016, p. 534

57.MATEI, M., SANDU, I., BIRSA, M.L., SARBU, L.G., SIMION, L., Rev. Chim. (Bucharest), 68, no. 1, 2017, p. 81.

58.SARBU, L.G., BAHRIN, L.G., JONES, P.G., BIRSA, M.L., HOPF, H., Beilstein J. Org. Chem., 11, 2015, p. 1917.

59. LUNGU, N.C., SANDU, I., SARBU, L.G., Rev. Chim. (Bucharest), 69, no. 12,2018 , p. 3549.

60. CIOBANU, A.S., GOANTA, M., BIRSA, A., ASAFTEI, I.V., BIRSA, M.L., Acta Chem. Iasi, 16, 2008, p. 61.

61.BRAVERMAN, S., CHERKINSKY, M., BIRSA, M.L., Science of Synthesis, 18.2, Georg Thieme Verlag, Stuttgart, 2005, p 55.

62.BIRSA, M.L., Synth. Commun., 31, 2001, p. 1271.

63.BIRSA, M. L., Sulfur Lett, 26, 2003, p. 155. 